TRANSACTIONS OF THE

AMERICAN MATHEMATICAL SOCIETY

Volume 353, Number 10, Pages 4189-4202

S 0002-9947(01)02851-3

Article electronically published on June 1, 2001

\title{
INVARIANT DISTRIBUTIONS SUPPORTED ON THE NILPOTENT CONE OF A SEMISIMPLE LIE ALGEBRA
}

\author{
THIERRY LEVASSEUR
}

\begin{abstract}
Let $\mathfrak{g}$ be a semisimple complex Lie algebra with adjoint group $G$ and $\mathcal{D}(\mathfrak{g})$ be the algebra of differential operators with polynomial coefficients on $\mathfrak{g}$. If $\mathfrak{g}_{0}$ is a real form of $\mathfrak{g}$, we give the decomposition of the semisimple $\mathcal{D}(\mathfrak{g})^{G}$-module of invariant distributions on $\mathfrak{g}_{0}$ supported on the nilpotent cone.
\end{abstract}

\section{INTRODUCTION}

Let $\mathfrak{g}$ be a semisimple complex Lie algebra with adjoint group $G$. Choose a Cartan subalgebra $\mathfrak{h}$ of $\mathfrak{g}$ and let $W$ be the associated Weyl group. Denote by $W^{\wedge}$ the set of isomorphism classes of irreducible $W$-modules and by $\mathcal{H}\left(\mathfrak{h}^{*}\right)$ the graded vector space of $W$-harmonic polynomials on $\mathfrak{h}$. For $\chi \in W^{\wedge}$, set

$$
b(\chi)=\inf \left\{j \in \mathbb{N}:\left[\mathcal{H}^{j}\left(\mathfrak{h}^{*}\right): \chi\right] \neq 0\right\}
$$

and choose a $W$-submodule $V_{\chi} \subset \mathcal{H}^{b(\chi)}\left(\mathfrak{h}^{*}\right)$ in the class of $\chi$. Denote by $d(\chi)$ the dimension of $V_{\chi}$.

Let $S\left(\mathfrak{g}^{*}\right)$ be the algebra of polynomial functions on $\mathfrak{g}$ and $\mathcal{D}(\mathfrak{g})$ be the algebra of differential operators on $\mathfrak{g}$, with coefficients in $S\left(\mathfrak{g}^{*}\right)$. The group $G$ acts on $\mathfrak{g}$, via the adjoint action, and hence has an induced action on $S\left(\mathfrak{g}^{*}\right), S(\mathfrak{g})$ and $\mathcal{D}(\mathfrak{g})$. Denote the differential of this action by $\tau: \mathfrak{g} \rightarrow \mathcal{D}(\mathfrak{g})$. Let $S_{+}(\mathfrak{g})^{G}$ and $S_{+}\left(\mathfrak{g}^{*}\right)^{G}$ be the set of invariant elements without constant term. Recall that $\mathbf{N}(\mathfrak{g})$, the nilpotent cone of $\mathfrak{g}$, is the variety of zeroes of the ideal $S_{+}\left(\mathfrak{g}^{*}\right)^{G} S\left(\mathfrak{g}^{*}\right)$.

Let $\mathfrak{g}_{0}$ be a real form of $\mathfrak{g}$ with adjoint group $G_{0} \subset G$. Denote by $\operatorname{Db}\left(\mathfrak{g}_{0}\right)$ the $\mathcal{D}(\mathfrak{g})$-module of distributions on $\mathfrak{g}_{0}$. Then, the subspace of invariant distributions $\mathrm{Db}\left(\mathfrak{g}_{0}\right)^{G_{0}}=\left\{T \in \operatorname{Db}\left(\mathfrak{g}_{0}\right): \tau(\mathfrak{g}) \cdot T=0\right\}$ is a $\mathcal{D}(\mathfrak{g})^{G}$-module, containing the submodule of invariant distributions supported on the nilpotent cone

$$
\mathrm{Db}\left(\mathfrak{g}_{0}\right)_{\text {nil }}^{G_{0}}=\left\{\Theta \in \mathrm{Db}\left(\mathfrak{g}_{0}\right)^{G_{0}}: \operatorname{Supp} \Theta \subset \mathbf{N}\left(\mathfrak{g}_{0}\right)\right\}
$$

where $\mathbf{N}\left(\mathfrak{g}_{0}\right)=\mathbf{N}(\mathfrak{g}) \cap \mathfrak{g}_{0}$ is the nilpotent cone of $\mathfrak{g}_{0}$. The structure of $\operatorname{Db}\left(\mathfrak{g}_{0}\right)_{\text {iil }}^{G_{0}}$ as a vector space is well understood, see, for example, [1,5]. Let $\left[\mathfrak{h}_{1}\right], \ldots,\left[\mathfrak{h}_{r}\right]$ be the conjugacy classes of Cartan subalgebras of $\mathfrak{g}_{0}$. For each $j$, let $\varepsilon_{I, j}: W\left(\mathfrak{h}_{j}\right) \rightarrow\{ \pm 1\}$ be

Received by the editors November 17, 1998.

1991 Mathematics Subject Classification. Primary 14L30, 16S32, 17B20, 22 E46.

Key words and phrases. Semisimple Lie algebra, invariant distribution, nilpotent orbit, Weyl group representation.

Research partially supported by EC TMR network "Algebraic Lie Representations", Grant No. ERB FMRX-CT97-0100. 
the imaginary signature of the real Weyl group $W\left(\mathfrak{h}_{j}\right)$. Then [5] Proposition 6.1.1] there exists a vector space isomorphism

$$
\bigoplus_{j=1}^{r} S\left(\mathfrak{h}_{j, \mathbb{C}}\right)^{\varepsilon_{I, j}} \stackrel{\sim}{\longrightarrow} \mathrm{Db}\left(\mathfrak{g}_{0}\right)_{n i l}^{G_{0}}
$$

where $S\left(\mathfrak{h}_{j, \mathbb{C}}\right)^{\varepsilon_{I, j}}$ is the isotypic component of type $\varepsilon_{I, j}$ in the $W\left(\mathfrak{h}_{j}\right)$-module $S\left(\mathfrak{h}_{j, \mathbb{C}}\right)$.

One aim of this note is to give a complete description of the $\mathcal{D}(\mathfrak{g})^{G}$-module $\operatorname{Db}\left(\mathfrak{g}_{0}\right)_{n i l}^{G_{0}}$. This description is given in terms of the simple summands of the equivariant holonomic $\mathcal{D}(\mathfrak{g})$-module

$$
\mathcal{M}=\mathcal{D}(\mathfrak{g}) /\left(\mathcal{D}(\mathfrak{g}) \tau(\mathfrak{g})+\mathcal{D}(\mathfrak{g}) S_{+}\left(\mathfrak{g}^{*}\right)^{G}\right) .
$$

By [9], [18] or [13], it is known that we have a decomposition

$$
\mathcal{M}=\bigoplus_{\chi \in W^{\wedge}} d(\chi) \mathcal{M}_{\chi}
$$

where the $\mathcal{M}_{\chi}$ are pairwise non-isomorphic simple $\mathcal{D}(\mathfrak{g})$-modules. Moreover, the support (in $\mathfrak{g}$ ) of $\mathcal{M}_{\chi}$ is the closure of a nilpotent orbit and $\mathcal{M}_{\chi}^{G}$ is a simple $\mathcal{D}(\mathfrak{g})^{G}$ module. Then we have, see Corollary 3.6 .

Theorem A. The $\mathcal{D}(\mathfrak{g})^{G}$-module $\operatorname{Db}\left(\mathfrak{g}_{0}\right)_{\text {nil }}^{G_{0}}$ decomposes as

$$
\mathrm{Db}\left(\mathfrak{g}_{0}\right)_{n i l}^{G_{0}} \cong \bigoplus_{\chi \in W^{\wedge}} m_{\chi} \mathcal{M}_{\chi}^{G}
$$

where $m_{\chi}=\sum_{j=1}^{r} \operatorname{dim} V_{\chi}^{\varepsilon_{I, j}}$.

This theorem is proved by combining the isomorphism $(*)$ and the properties, established in 18, 11, 12, 13], of the Harish-Chandra homomorphism

$$
\delta: \mathcal{D}(\mathfrak{g})^{G} \longrightarrow \mathcal{D}(\mathfrak{h})^{W} .
$$

In the particular case where $\mathfrak{g}_{0}$ is a complex Lie algebra $\mathfrak{g}_{1}$ (viewed as a real Lie algebra), Theorem $\mathrm{A}$ was proved by N. Wallach [18. In this case, $\mathfrak{g} \simeq \mathfrak{g}_{1} \times \mathfrak{g}_{1}$, $W \simeq W_{1} \times W_{1}$ where $W_{1}$ is the Weyl group of $\mathfrak{g}_{1}$. Then, each $\mathcal{M}_{\chi}$ occurring in the decomposition of $\mathrm{Db}\left(\mathfrak{g}_{0}\right)_{n i l}^{G_{0}}$ is of the form $\mathcal{M}_{\phi} \otimes \mathcal{M}_{\phi}$ with $\chi=\phi \bigotimes \phi, \phi \in W_{1}^{\widehat{1}}$, and one has $m_{\chi}=1$. Hence $\operatorname{Db}\left(\mathfrak{g}_{0}\right)_{\text {nil }}^{G_{0}} \cong \bigoplus_{\phi \in W_{1}^{\wedge}} \mathcal{M}_{\phi}^{G_{1}} \otimes \mathcal{M}_{\phi}^{G_{1}}$ as a $\mathcal{D}(\mathfrak{g})^{G}$-module.

The next corollary is an easy consequence of Theorem $\mathrm{A}$

Corollary B. Let $\chi \in W^{\wedge}$. then, $\mathcal{M}_{\chi} \cong \mathcal{D}(\mathfrak{g})$. $\Theta$ for some $\Theta \in \operatorname{Db}\left(\mathfrak{g}_{0}\right)$ if, and only if, $V_{\chi}^{\varepsilon_{I, j}} \neq 0$ for some $j \in\{1, \ldots, r\}$.

In Remark [3.7] we apply this result to give examples of modules $\mathcal{M}_{\chi}$ which cannot be generated by a distribution on any real form of $\mathfrak{g}$.

\section{Preliminary Results}

We retain the notation of the introduction. Denote by $\Delta$ the root system of $\mathfrak{h}$ in $\mathfrak{g}$ and fix a system $\Delta^{+}$of positive roots. Set $n=\operatorname{dim} \mathfrak{g}, \ell=\operatorname{dim} \mathfrak{h}$ and $\nu=\# \Delta^{+}$, hence $n=2 \nu+\ell$. Let $\pi$ be the product of positive roots and recall that $x \in \mathfrak{g}$ is called generic if $\pi(x) \neq 0$. If $\mathfrak{a} \subset \mathfrak{g}$, we denote by $\mathfrak{a}^{\prime}$ the set of generic elements in $\mathfrak{a}$.

For $q \in S(\mathfrak{g})$, let $\partial(q) \in \mathcal{D}(\mathfrak{g})$ be the corresponding differential operator with constant coefficients. Let $\left\{e_{i}\right\}_{1 \leqslant i \leqslant n}$ be an orthonormal basis of $\mathfrak{g}$ with respect to the Killing form $\kappa$ such that $\left\{e_{i}\right\}_{1 \leqslant i \leqslant \ell}$ is a basis of $\mathfrak{h}$. Denote by $x_{i} \in S\left(\mathfrak{g}^{*}\right)$, 
$1 \leqslant i \leqslant n$, the associated coordinate functions; thus $\partial\left(e_{i}\right)$ identifies with the partial derivative $\partial_{i}=\frac{\partial}{\partial x_{i}}$. Denote the Euler vector fields on $\mathfrak{g}$ and $\mathfrak{h}$ by $\mathbb{E}_{\mathfrak{g}}=\sum_{i=1}^{n} x_{i} \partial_{i}$ and $\mathrm{E}_{\mathfrak{h}}=\sum_{i=1}^{\ell} x_{i} \partial_{i}$.

We now give some notation and results from [11, 12, 13, 18. Recall first that the algebra homomorphism, defined by Harish-Chandra,

$$
\delta: \mathcal{D}(\mathfrak{g})^{G} \longrightarrow \mathcal{D}(\mathfrak{h})^{W}
$$

extends the Chevalley isomorphisms $S(\mathfrak{g})^{G} \stackrel{\sim}{\longrightarrow} S(\mathfrak{h})^{W}$ and $S\left(\mathfrak{g}^{*}\right)^{G} \stackrel{\sim}{\longrightarrow} S\left(\mathfrak{h}^{*}\right)^{W}$. The map $\delta$ is surjective and its kernel is $\mathcal{I}=(\mathcal{D}(\mathfrak{g}) \tau(\mathfrak{g}))^{G}$. This enables one to identify, through $\delta$, modules over $A(\mathfrak{g}):=\mathcal{D}(\mathfrak{g})^{G} / \mathcal{I}$ with $\mathcal{D}(\mathfrak{h})^{W}$-modules.

Lemma 1.1. Let $D \in \mathcal{D}(\mathfrak{g})^{G}$. Then $D=P+Q$ with $P \in \mathbb{C}\left\langle S(\mathfrak{g})^{G}, S\left(\mathfrak{g}^{*}\right)^{G}\right\rangle$ and $Q \in \mathcal{I}$.

Proof. By [11, we know that $\mathcal{D}(\mathfrak{h})^{W}=\mathbb{C}\left\langle S(\mathfrak{h})^{W}, S\left(\mathfrak{h}^{*}\right)^{W}\right\rangle$. The lemma is therefore a consequence of the properties of $\delta$ previously recalled.

Recall that the $\left(\mathcal{D}(\mathfrak{h})^{W}, W\right)$-module $S\left(\mathfrak{h}^{*}\right)$ decomposes as

$$
S\left(\mathfrak{h}^{*}\right) \cong \bigoplus_{\chi \in W^{\wedge}} V^{\chi} \otimes_{\mathbb{C}} V_{\chi}
$$

where $V^{\chi}=\operatorname{Hom}_{W}\left(V_{\chi}, S\left(\mathfrak{h}^{*}\right)\right)$ is a simple $\mathcal{D}(\mathfrak{h})^{W}$-module. Let $\left\{v_{\chi}^{1}, \ldots, v_{\chi}^{d(\chi)}\right\}$ be a basis of $V_{\chi}$, then $V^{\chi} \cong \mathcal{D}(\mathfrak{h})^{W} \cdot v_{\chi}^{j}$ for all $j$ and (1.1) implies that

$$
S\left(\mathfrak{h}^{*}\right)=\bigoplus_{\chi \in W^{\wedge}} \bigoplus_{j=1}^{d(\chi)} \mathcal{D}(\mathfrak{h})^{W} . v_{\chi}^{j} .
$$

Now, set $\mathcal{N}=\mathcal{D}(\mathfrak{g}) / \mathcal{D}(\mathfrak{g}) \tau(\mathfrak{g}) \otimes_{A(\mathfrak{g})} S\left(\mathfrak{h}^{*}\right)$ and $\mathcal{N}_{\chi}=\mathcal{D}(\mathfrak{g}) / \mathcal{D}(\mathfrak{g}) \tau(\mathfrak{g}) \otimes_{A(\mathfrak{g})} V^{\chi}$. We have

$$
\mathcal{N}=\mathcal{D}(\mathfrak{g}) /\left(\mathcal{D}(\mathfrak{g}) \tau(\mathfrak{g})+\mathcal{D}(\mathfrak{g}) S_{+}(\mathfrak{g})^{G}\right)
$$

and, using (1.1),

$$
\mathcal{N}=\bigoplus_{\chi \in W^{\wedge}} \mathcal{N}_{\chi} \otimes_{\mathbb{C}} V_{\chi}
$$

Then each $\mathcal{N}_{\chi}$ is a simple (holonomic) $\mathcal{D}(\mathfrak{g})$-module 13 and, therefore, $\mathcal{N}$ is a semisimple $\mathcal{D}(\mathfrak{g})$-module (see also $[9]$ ). Let $\mathcal{C}(\mathcal{N})$ be the full subcategory of finitely generated $\mathcal{D}(\mathfrak{g})$-modules of the form $\bigoplus_{\chi \in W^{\wedge}} m_{\chi} \mathcal{N}_{\chi}, m_{\chi} \in \mathbb{N}$. From [13] we know that the category $\mathrm{C}(\mathcal{N})$ is equivalent to the category $W$-mod (of finite dimensional $W$-modules) via the functor

$$
\text { Sol : C }(\mathcal{N}) \longrightarrow W \text {-mod, } \quad \operatorname{Sol}(N)=\operatorname{Hom}_{\mathcal{D}(\mathfrak{h})^{W}}\left(N^{G}, S\left(\mathfrak{h}^{*}\right)\right)
$$

where $W$ acts on $\operatorname{Sol}(N)$ through its natural action on $S\left(\mathfrak{h}^{*}\right)$.

The Killing form $\kappa$ induces a $G$-isomorphism $\mathfrak{g} \stackrel{\sim}{\sim} \mathfrak{g}^{*}$ and an algebra automorphism $\varkappa$ of $\mathcal{D}(\mathfrak{g})$, defined by $\varkappa(\partial(v))=\kappa\left(v,_{-}\right), \varkappa\left(\kappa\left(v,,_{-}\right)\right)=-\partial(v)$, for all $v \in \mathfrak{g}$. Hence, in coordinates, $\varkappa\left(\partial_{j}\right)=x_{j}, \varkappa\left(x_{j}\right)=-\partial_{j}$. Set $i=\sqrt{-1} \in \mathbb{C}$ and denote by $i$ the automorphism of $\mathcal{D}(\mathfrak{g})$ given by $i\left(\partial_{j}\right)=-i \partial_{j}$, $\mathbf{i}\left(x_{j}\right)=i x_{j}$. Define then the "Fourier transformation" $F_{\mathfrak{g}} \in \operatorname{Aut} \mathcal{D}(\mathfrak{g})$ by $F_{\mathfrak{g}}=\mathrm{i} \circ \varkappa=\varkappa \circ \mathrm{i}^{-1}$; thus $F_{\mathfrak{g}}\left(x_{j}\right)=i \partial_{j}, F_{\mathfrak{g}}\left(\partial_{j}\right)=i x_{j}$. One easily checks that $\varkappa(\tau(x))=F_{\mathfrak{g}}(\tau(x))=\tau(x)$ 
for all $x \in \mathfrak{g}$; moreover, $\varkappa$ and $F_{\mathfrak{g}}$ are $G$-equivariant. Similarly, since $\kappa$ is nondegenerate and $W$-invariant on $\mathfrak{h}$, one can define $W$-equivariant automorphisms $\varkappa$ and $F_{\mathfrak{h}}=$ i $\circ \varkappa$ in $\operatorname{Aut} \mathcal{D}(\mathfrak{h})$.

Lemma 1.2. One has $\delta \circ F_{\mathfrak{g}}=F_{\mathfrak{h}} \circ \delta$.

Proof. A direct computation shows that $\delta\left(F_{\mathfrak{g}}(P)\right)=F_{\mathfrak{h}}(\delta(P))$ when $P$ belongs to $S(\mathfrak{g})^{G}$ or $S\left(\mathfrak{g}^{*}\right)^{G}$. Since $\delta$ is a homomorphism, it follows that $\delta\left(F_{\mathfrak{g}}(P)\right)=F_{\mathfrak{h}}(\delta(P))$ for all $P \in \mathbb{C}\left\langle S(\mathfrak{g})^{G}, S\left(\mathfrak{g}^{*}\right)^{G}\right\rangle$. Now, let $D \in \mathcal{D}(\mathfrak{g})^{G}$ and write $D=P+Q$ as in Lemma 1.1. Then, since $F_{\mathfrak{g}}(\mathcal{I})=\mathcal{I}$, we have $\delta\left(F_{\mathfrak{g}}(D)\right)=\delta\left(F_{\mathfrak{g}}(P)\right)=F_{\mathfrak{h}}(\delta(P))=$ $F_{\mathfrak{h}}(\delta(D))$.

Recall that $\mathcal{H}\left(\mathfrak{h}^{*}\right)$ is the vector space of $W$-harmonic polynomials on $\mathfrak{h}$. Hence

$$
\mathcal{H}\left(\mathfrak{h}^{*}\right)=\left\{f \in S\left(\mathfrak{h}^{*}\right): \partial(q) . f=0 \text { for all } q \in S_{+}(\mathfrak{h})^{W}\right\}
$$

and, as a $W$-module, $\mathcal{H}\left(\mathfrak{h}^{*}\right)$ identifies with the regular representation of $W$. The vector space $\mathcal{H}\left(\mathfrak{h}^{*}\right)$ is a graded subspace of $S\left(\mathfrak{h}^{*}\right)$ and we set $\mathcal{H}^{j}\left(\mathfrak{h}^{*}\right)=S^{j}\left(\mathfrak{h}^{*}\right) \cap$ $\mathcal{H}\left(\mathfrak{h}^{*}\right), 0 \leqslant j \leqslant \nu$. Define the harmonic elements of $S(\mathfrak{h})$ by $\mathcal{H}(\mathfrak{h})=F_{\mathfrak{h}}\left(\mathcal{H}\left(\mathfrak{h}^{*}\right)\right)=$ $\bigoplus_{j=0}^{\nu} \mathcal{H}^{j}(\mathfrak{h})$. (We could as well have set $\mathcal{H}(\mathfrak{h})=\varkappa\left(\mathcal{H}\left(\mathfrak{h}^{*}\right)\right.$ ), since $\mathcal{H}^{j}\left(\mathfrak{h}^{*}\right)$ is stable under i.)

Since $V_{\chi} \subset \mathcal{H}^{b(\chi)}\left(\mathfrak{h}^{*}\right)$, we have $\left(\mathrm{E}_{\mathfrak{h}}-b(\chi)\right) \cdot v_{\chi}^{j}=0$. For all $d \in L:=\operatorname{ann}_{\mathcal{D}(\mathfrak{h})^{W}}\left(v_{\chi}^{j}\right)$, we have $\left[\mathrm{E}_{\mathfrak{h}}-b(\chi), d\right]=\left[\mathrm{E}_{\mathfrak{h}}, d\right] \in L$. It follows that $L=\bigoplus_{k \in \mathbb{Z}} L \cap \mathcal{D}^{k}(\mathfrak{h})^{W}$, where $\mathcal{D}^{k}(\mathfrak{h})=\left\{d \in \mathcal{D}(\mathfrak{h}):\left[\mathrm{E}_{\mathfrak{h}}, d\right]=k d\right\}$. Equivalently, $L$ is stable under the $\mathbb{C}^{*}$-action on $\mathcal{D}(\mathfrak{h})$ given by $f \mapsto \lambda f, \partial(v) \mapsto \lambda^{-1} \partial(v), f \in \mathfrak{h}^{*}, v \in \mathfrak{h}$. In particular, we see that $F_{\mathfrak{h}}(L)=\varkappa(L)$.

Let $R$ be a ring and $\alpha \in \operatorname{Aut}(R)$. If $M$ is an $R$-module, we define the $R$-module $M^{\alpha}$ to be the abelian group $M$ with action of $a \in R$ on $x \in M$ given by $a \cdot x=\alpha(a) x$. This applies to the modules $\mathcal{N}, \mathcal{N}_{\chi}$ and the automorphism $\alpha=F_{\mathfrak{g}}{ }^{-1}$. Define

$$
\mathcal{M}=\mathcal{N}^{F_{\mathfrak{g}}^{-1}}, \quad \mathcal{M}_{\chi}=\mathcal{N}_{\chi}^{F_{\mathfrak{g}}^{-1}} .
$$

Thus, from (1.2) and (1.3), we obtain

$$
\mathcal{M}=\mathcal{D}(\mathfrak{g}) /\left(\mathcal{D}(\mathfrak{g}) \tau(\mathfrak{g})+\mathcal{D}(\mathfrak{g}) S_{+}\left(\mathfrak{g}^{*}\right)^{G}\right) \cong \bigoplus_{\chi \in W^{\wedge}} \mathcal{M}_{\chi} \otimes_{\mathbb{C}} V_{\chi}
$$

Remark. In 13] one defines $\mathcal{M}_{\chi}$ to be $\mathcal{N}_{\chi}^{\varkappa^{-1}}$, but the two definitions agree. Indeed, let $V^{\chi} \cong \mathcal{D}(\mathfrak{h})^{W} . v_{\chi}^{j}=\mathcal{D}(\mathfrak{h})^{W} / L$ be as above. Then,

$$
\mathcal{N}_{\chi} \cong \mathcal{D}(\mathfrak{g}) / J, \quad J=\mathcal{D}(\mathfrak{g}) \tau(\mathfrak{g})+\mathcal{D}(\mathfrak{g}) S_{+}(\mathfrak{g})^{G}+\mathcal{D}(\mathfrak{g}) \delta^{-1}(L) .
$$

Write $\mathcal{N}_{\chi}=\mathcal{D}(\mathfrak{g}) \cdot\left(\overline{1} \otimes_{A(\mathfrak{g})} v_{\chi}^{j}\right)$, where $\overline{1}$ is the canonical generator of $\mathcal{D}(\mathfrak{g}) / \mathcal{D}(\mathfrak{g}) \tau(\mathfrak{g})$. From $\delta\left(\mathrm{E}_{\mathfrak{g}}\right)=\mathrm{E}_{\mathfrak{h}}-\nu$, we get that $\left(\mathrm{E}_{\mathfrak{g}}-(b(\chi)-\nu)\right) \cdot\left(\overline{1} \otimes_{A(\mathfrak{g})} v_{\chi}^{j}\right)=0$. It follows (as above) that $J$ is stable under the natural $\mathbb{C}^{*}$-action on $\mathcal{D}(\mathfrak{g})$. Hence, $F_{\mathfrak{g}}(J)=\varkappa(J)$ and we have $\mathcal{N}_{\chi}^{\varkappa^{-1}}=\mathcal{N}_{\chi}^{F_{\mathfrak{g}}^{-1}}$.

We can define the category $\mathrm{C}(\mathcal{M})$ similar to $\mathrm{C}(\mathcal{N})$. We clearly have $M \in \mathrm{C}(\mathcal{N})$ if, and only if, $N=M^{F_{\mathfrak{g}}} \in \mathrm{C}(\mathcal{N})$. Moreover, by [13], this is equivalent to saying that $M$ is a $G$-equivariant finitely generated $\mathcal{D}(\mathfrak{g})$-module such that $M=\mathcal{D}(\mathfrak{g}) M^{G}$ and Supp $M \subset \mathbf{N}(\mathfrak{g})$. This is also equivalent to: $N$ is a $G$-equivariant finitely generated $\mathcal{D}(\mathfrak{g})$-module such that $N=\mathcal{D}(\mathfrak{g}) N^{G}$ and $N$ is $S_{+}$-finite (meaning that each $v \in N$ is killed by a power of $\left.S_{+}(\mathfrak{g})^{G}\right)$.

Recall that $\mathcal{N}_{\chi}^{G} \simeq V^{\chi}$ through the identification of $A(\mathfrak{g})$ with $\mathcal{D}(\mathfrak{h})^{W}$. 
Lemma 1.3. One has $\mathcal{M}_{\chi}^{G} \stackrel{\sim}{\longrightarrow}\left(V^{\chi}\right)^{F_{\mathfrak{h}}{ }^{-1}}$.

Proof. Write $\mathcal{N}_{\chi}=\mathcal{D}(\mathfrak{g}) / J$. Then, $\mathcal{M}_{\chi}=\mathcal{D}(\mathfrak{g}) / F_{\mathfrak{g}}(J)$ and $\mathcal{M}_{\chi}^{G}=\mathcal{D}(\mathfrak{g})^{G} / F_{\mathfrak{g}}\left(J^{G}\right)$. By Lemma $1.2, \delta\left(F_{\mathfrak{g}}\left(J^{G}\right)\right)=F_{\mathfrak{h}}\left(\delta\left(J^{G}\right)\right)$, therefore $\mathcal{M}_{\chi}^{G} \stackrel{\sim}{\sim} \mathcal{D}(\mathfrak{h})^{W} / F_{\mathfrak{h}}\left(\delta\left(J^{G}\right)\right)$. Since $V^{\chi} \cong \mathcal{D}(\mathfrak{h})^{W} / \delta\left(J^{G}\right)$, the lemma follows.

Let $\mathfrak{g}_{0}$ be a real form of $\mathfrak{g}$ with adjoint group $G_{0} \subset G$. There exists a natural action of $\mathcal{D}(\mathfrak{g})$ on $\mathrm{Db}\left(\mathfrak{g}_{0}\right)$ defined by

$$
\langle\partial(v) . T, f\rangle=\langle T,-\partial(v) . f\rangle, \quad\langle\xi . T, f\rangle=\langle T, \xi f\rangle
$$

for all $T \in \operatorname{Db}\left(\mathfrak{g}_{0}\right), f \in \mathcal{C}_{c}^{\infty}\left(\mathfrak{g}_{0}\right), v \in \mathfrak{g}, \xi \in \mathfrak{g}^{*}$. This induces a structure of $\mathcal{D}(\mathfrak{g})^{G_{-}}$ module on $\operatorname{Db}\left(\mathfrak{g}_{0}\right)^{G_{0}}$. From $\mathcal{I} \cdot \operatorname{Db}\left(\mathfrak{g}_{0}\right)^{G_{0}}=0$, we obtain a natural $A(\mathfrak{g})$-module structure on $\operatorname{Db}\left(\mathfrak{g}_{0}\right)^{G_{0}}$.

Fix a basis $\left\{u_{1}, \ldots, u_{n}\right\}$ of $\mathfrak{g}_{0}$ such that $\kappa\left(u_{j}, u_{k}\right)= \pm \delta_{j k}$ and denote by $d y$ the Lebesgue measure associated to this choice. Let $\mathcal{S}\left(\mathfrak{g}_{0}\right)$ be the Schwartz space on $\mathfrak{g}_{0}$. Define, as in [18, Appendix 1], the Fourier transform of $f \in \mathcal{S}\left(\mathfrak{g}_{0}\right)$ by

$$
\hat{f}(x)=\frac{1}{(2 \pi)^{n / 2}} \int_{\mathfrak{g}_{0}} f(y) e^{-i \kappa(y, x)} d y .
$$

Let $T$ be a tempered distribution on $\mathfrak{g}_{0}$. The Fourier transform of $T$ is defined by $\langle\widehat{T}, f\rangle=\langle T, \hat{f}\rangle$ for $f \in \mathcal{C}_{c}^{\infty}\left(\mathfrak{g}_{0}\right)$. Then we have

$$
\forall D \in \mathcal{D}(\mathfrak{g}), \quad \forall T \in \mathrm{Db}\left(\mathfrak{g}_{0}\right), \quad \widehat{D . T}=F_{\mathfrak{g}}(D) . \widehat{T} .
$$

Recall [2] that $T \in \mathrm{Db}\left(\mathfrak{g}_{0}\right)$ is said to be homogeneous of degree $d$ if, for all $f \in$ $\mathcal{C}_{c}^{\infty}\left(\mathfrak{g}_{0}\right), t \in \mathbb{R}^{*},\left\langle T, f_{t}\right\rangle=t^{d}\langle T, f\rangle$, where $f_{t}(v)=t^{-n} f\left(t^{-1} v\right)$. Then, a homogeneous distribution of degree $d$ is tempered and satisfies $\mathrm{E}_{\mathfrak{g}} \cdot T=d T$. We will need the following well-known result:

Lemma 1.4. Let $T \in \mathrm{Db}\left(\mathfrak{g}_{0}\right)$ be tempered and set $M=\mathcal{D}(\mathfrak{g}) \cdot T$. Then $M^{F_{\mathfrak{g}} \cong}$ $\mathcal{D}(\mathfrak{g}) \cdot \widehat{T}$.

Proof. By (1.4) we have $\operatorname{ann}_{\mathcal{D}}(\mathfrak{g})(\widehat{T})=F_{\mathfrak{g}}{ }^{-1}\left(\operatorname{ann}_{\mathcal{D}}(\mathfrak{g})(T)\right)$. Hence the result.

Let $\mathbf{N}\left(\mathfrak{g}_{0}\right)$ be the set of nilpotent elements of $\mathfrak{g}_{0}$. Define $\mathcal{D}(\mathfrak{g})$-submodules of $\mathrm{Db}\left(\mathfrak{g}_{0}\right)$ by

$$
\begin{aligned}
& \mathrm{Db}\left(\mathfrak{g}_{0}\right)_{n i l}=\left\{\Theta \in \mathrm{Db}\left(\mathfrak{g}_{0}\right): \operatorname{Supp} \Theta \subset \mathbf{N}\left(\mathfrak{g}_{0}\right)\right\} \\
& \operatorname{Db}\left(\mathfrak{g}_{0}\right)_{S_{+}}=\left\{T \in \operatorname{Db}\left(\mathfrak{g}_{0}\right): \exists k \in \mathbb{N}, S_{+}(\mathfrak{g})^{k} \cdot T=0\right\}
\end{aligned}
$$

The elements of $\mathrm{Db}\left(\mathfrak{g}_{0}\right)_{S_{+}}$are called $S_{+}$-finite. Observe that $\mathrm{Db}\left(\mathfrak{g}_{0}\right)_{\text {nil }}^{G_{0}}$ and $\mathrm{Db}\left(\mathfrak{g}_{0}\right)_{S_{+}}^{G_{0}}$ are $\mathcal{D}(\mathfrak{g})^{G}$-modules. The next theorem is a consequence of the results proved in 18 .

Theorem 1.5. (1) $\mathrm{Db}\left(\mathfrak{g}_{0}\right)_{\text {nil }}^{G_{0}}=\left\{\Theta \in \mathrm{Db}\left(\mathfrak{g}_{0}\right)^{G_{0}}: \mathcal{D}(\mathfrak{g}) \cdot \Theta \in \mathrm{C}(\mathcal{M})\right\}$.

(2) $\mathrm{Db}\left(\mathfrak{g}_{0}\right)_{S_{+}}^{G_{0}}=\left\{T \in \mathrm{Db}\left(\mathfrak{g}_{0}\right)^{G_{0}}: \mathcal{D}(\mathfrak{g}) \cdot T \in \mathrm{C}(\mathcal{N})\right\}$.

(3) $\Theta \in \mathrm{Db}\left(\mathfrak{g}_{0}\right)_{n i l}^{G_{0}} \Longleftrightarrow \widehat{\Theta} \in \mathrm{Db}\left(\mathfrak{g}_{0}\right)_{S_{+}}^{G_{0}}$.

Proof. (1) follows from [18, Theorem 6.1], since $\mathcal{D}(\mathfrak{g}) \cdot \Theta \in \mathrm{C}(\mathcal{M})$ is equivalent to $\mathcal{D}(\mathfrak{g})^{G} . \Theta \cong \bigoplus_{\chi \in W^{\wedge}} m_{\chi} \mathcal{M}_{\chi}^{G}$.

(2) and (3) are consequences of (1) and Lemma 1.4 
Remark 1.6. Let $T \in \mathrm{Db}\left(\mathfrak{g}_{0}\right)_{S_{+}}^{G_{0}}$. Recall that by the Harish-Chandra regularity theorem, $T$ is given by

$$
\langle T, f\rangle=\int_{\mathfrak{g}_{0}^{\prime}} F_{T}(y) f(y) d y
$$

for some analytic function $F_{T}$ on $\mathfrak{g}_{0}^{\prime}$, locally integrable on $\mathfrak{g}_{0}$.

\section{The distributions $\Theta_{u, \Gamma}$ And $T_{p, \Gamma}$}

Let $\mathfrak{g}_{0}$ be a real form of $\mathfrak{g}$, with adjoint group $G_{0}, \mathfrak{h}_{0}$ a Cartan subalgebra and let $H_{0}$ be the associated Cartan subgroup. Set $\mathfrak{h}=\mathbb{C} \otimes_{\mathbb{R}} \mathfrak{h}_{0}$ and adopt the notation of \$1. Denote by $W\left(\mathfrak{h}_{0}\right)$ the real Weyl group, i.e. $W\left(\mathfrak{h}_{0}\right)=N_{G_{0}}\left(\mathfrak{h}_{0}\right) / Z_{G_{0}}\left(\mathfrak{h}_{0}\right)$. Define

$$
\begin{aligned}
\Delta_{R} & =\left\{\alpha \in \Delta: \alpha\left(\mathfrak{h}_{0}\right) \subset \mathbb{R}\right\} \quad \text { (the real roots), } \\
\Delta_{I} & =\left\{\alpha \in \Delta: \alpha\left(\mathfrak{h}_{0}\right) \subset i \mathbb{R}\right\} \quad \text { (the imaginary roots). }
\end{aligned}
$$

A root which is neither real nor imaginary is called complex. Let $\Delta_{I}^{+}$be a positive system of roots in $\Delta_{I}$ and set $\pi_{I}=\prod_{\alpha \in \Delta_{I}^{+}} \alpha$. Then each $w \in W\left(\mathfrak{h}_{0}\right)$ permutes the imaginary roots and one can define a character of $W\left(\mathfrak{h}_{0}\right)$, the imaginary signature, by

$$
\varepsilon_{I}: W\left(\mathfrak{h}_{0}\right) \rightarrow\{ \pm 1\}, \quad w \cdot \pi_{I}=\varepsilon_{I}(w) \pi_{I} .
$$

If $V$ is a $W\left(\mathfrak{h}_{0}\right)$-module we denote by $V^{\varepsilon_{I}}$ the isotypic component of type $\varepsilon_{I}$ in $V$.

In the sequel, we adopt the notation of [5] with the minor difference that we use $e^{-i \kappa(x, y)}$ in the definition of the Fourier transform.

Let $h \in \mathfrak{h}_{0}^{\prime}$ and $f \in \mathcal{C}_{c}^{\infty}\left(\mathfrak{g}_{0}\right)$. Define [5, §3.1] the distribution $\mu_{G_{0} . h}$ by

$$
\left\langle\mu_{G_{0} . h}, f\right\rangle=\left|\operatorname{det} \operatorname{ad}_{\mathfrak{g}_{0} / \mathfrak{h}_{0}}(h)\right|^{\frac{1}{2}} \int_{G_{0} / H_{0}} f(\dot{g} \cdot h) d \dot{g} .
$$

Then one defines the function $J_{\mathfrak{g}_{0}}(f)$, or simply $J(f)$, on $\mathfrak{h}_{0}^{\prime}$ by

$$
J_{\mathfrak{g}_{0}}(f)=\left\{h \mapsto\left\langle\mu_{G_{0} . h}, f\right\rangle\right\} .
$$

Set $\mathfrak{h}_{0}^{\text {reg }}=\left\{h \in \mathfrak{h}_{0}: \pi_{I}(h) \neq 0\right\}$ and fix a connected component $\Gamma$ of $\mathfrak{h}_{0}^{\text {reg }}$. Let $u \in S(\mathfrak{h})$; Harish-Chandra has shown, see [17, §8.1, p. 123], that one can define a tempered $G_{0}$-invariant distribution on $\mathfrak{g}_{0}$ by

$$
\forall f \in \mathcal{C}_{c}^{\infty}\left(\mathfrak{g}_{0}\right), \quad\left\langle\Theta_{u, \Gamma}, f\right\rangle=\lim _{\substack{h \rightarrow 0 \\ h \in \Gamma}}[\partial(u) . J(f)](h) .
$$

Furthermore $\Theta_{u, \Gamma} \in \operatorname{Db}\left(\mathfrak{g}_{0}\right)_{n i l}^{G_{0}}$ and, when $u \in S^{b}(\mathfrak{h}), \Theta_{u, \Gamma}$ is homogeneous of degree $-b-\nu-\ell$.

Now let $p \in S\left(\mathfrak{h}^{*}\right)$ and define $T \in \mathrm{Db}\left(\mathfrak{g}_{0}\right)_{S_{+}}^{G_{0}}$ by

$$
T_{p, \Gamma}=\widehat{\Theta}_{F_{\mathfrak{h}}(p), \Gamma}=\left\{f \mapsto \lim _{\substack{h \rightarrow 0 \\ h \in \Gamma}}\left[\partial\left(F_{\mathfrak{h}}(p)\right) . J(\hat{f})\right](h)\right\} .
$$

Then, $T_{p, \Gamma}$ is tempered and is homogeneous of degree $b-\nu$ when $p \in S^{b}\left(\mathfrak{h}^{*}\right)$.

Lemma 2.1. (1) Let $\varphi \in S\left(\mathfrak{g}^{*}\right)^{G}$. Then, $\varphi T_{p, \Gamma}=T_{\delta(\varphi) p, \Gamma}$.

(2) Let $q \in S(\mathfrak{g})^{G}$. Then, $\partial(q) \cdot T_{p, \Gamma}=T_{\partial(\delta(q)) \cdot p, \Gamma}$. 
Proof. Set $u=F_{\mathfrak{h}}(p), \phi=\delta(\varphi) \in S\left(\mathfrak{h}^{*}\right)^{W}$ and $s=\delta(q) \in S(\mathfrak{h})^{W}$. Let $f \in \mathcal{C}_{c}^{\infty}\left(\mathfrak{g}_{0}\right)$.

(1) By definition, see (2.2), $\left\langle\varphi T_{p, \Gamma}, f\right\rangle=\lim _{h \rightarrow 0}[\partial(u) . J(\widehat{\varphi f})](h)$. But, [17] Lemma 3.2.7, p. 38], (1.4) and Lemma 1.2 imply that $J(\widehat{\varphi f})=\partial\left(F_{\mathfrak{h}}(\phi)\right) . J(\hat{f})$. Hence,

$$
\begin{aligned}
\left\langle\varphi T_{p, \Gamma}, f\right\rangle & =\lim _{\substack{h \rightarrow 0 \\
h \in \Gamma}}\left[\partial(u) \partial\left(F_{\mathfrak{h}}(\phi)\right) . J(\hat{f})\right](h)=\lim _{\substack{h \rightarrow 0 \\
h \in \Gamma}}\left[\partial\left(F_{\mathfrak{h}}(\phi p)\right) . J(\hat{f})\right](h) \\
& =\left\langle T_{\phi p, \Gamma}, f\right\rangle,
\end{aligned}
$$

as desired.

(2) By (1.4), $\partial(q) \cdot T_{p, \Gamma}$ is the Fourier transform of $F_{\mathfrak{g}}{ }^{-1}(q) \Theta_{u, \Gamma}$, hence

$$
\left\langle\partial(q) \cdot T_{p, \Gamma}, f\right\rangle=\lim _{\substack{h \rightarrow 0 \\ h \in \Gamma}}\left[\partial(u) . J\left(F_{\mathfrak{g}}{ }^{-1}(q) \hat{f}\right)\right](h) .
$$

Set $g=J(\hat{f})$. From [17, Lemma 3.2.7, p. 38] and Lemma [1.2 we obtain that $J\left(F_{\mathfrak{g}}{ }^{-1}(q) \hat{f}\right)={F_{\mathfrak{h}}}^{-1}(s) g$. Therefore

$$
\left\langle\partial(q) \cdot T_{p, \Gamma}, f\right\rangle=\lim _{\substack{h \rightarrow 0 \\ h \in \Gamma}}\left[\partial(u) \cdot\left(F_{\mathfrak{h}}{ }^{-1}(s) g\right)\right](h) .
$$

Recall (see \$1) that we have chosen a coordinate system $\left\{x_{j} ; e_{j}\right\}_{1 \leqslant j \leqslant \ell}$. With standard notation, we write $x^{\alpha}=\prod_{k=1}^{\ell} x_{k}^{\alpha_{k}}, e^{\mu}=\prod_{k=1}^{\ell} e_{k}^{\mu_{k}}$ and

$$
p=\sum_{\alpha \in \mathbb{N}^{\ell}} p_{\alpha} x^{\alpha}, \quad s=\sum_{\mu \in \mathbb{N}^{\ell}} s_{\mu} e^{\mu} .
$$

Set $\partial^{\mu}=\prod_{j} \partial\left(e_{j}\right)^{\mu_{j}}$; thus $\partial(s)=\sum_{\mu \in \mathbb{N}^{\ell}} s_{\mu} \partial^{\mu}$. Order $\mathbb{N}^{\ell}$ by saying that $\mu \leq \alpha$ if $\mu_{j} \leqslant \alpha_{j}$ for all $j$. Set $\alpha !=\prod_{j} \alpha_{j}$ ! and $\left(\begin{array}{l}\alpha \\ \mu\end{array}\right)=\prod_{j}\left(\begin{array}{l}\alpha_{j} \\ \mu_{j}\end{array}\right)$, when $\mu \leq \alpha$. Then,

$$
\partial^{\mu}\left(x^{\alpha}\right)= \begin{cases}0 & \text { if } \mu \not \leq \alpha, \\ \frac{\alpha !}{(\alpha-\mu) !} x^{\alpha-\mu} & \text { if } \mu \leq \alpha .\end{cases}
$$

Now we have $u=F_{\mathfrak{h}}(p)=\sum_{\alpha} p_{\alpha} i^{|\alpha|} \partial^{\alpha}$ and $F_{\mathfrak{h}}{ }^{-1}(s)=\sum_{\mu} q_{\mu} i^{-|\mu|} x^{\mu}$. Therefore, using the Leibniz formula, we get that

$$
\begin{aligned}
\partial(u) \cdot\left(F_{\mathfrak{h}}{ }^{-1}(s) g\right) & =\sum_{\alpha} p_{\alpha} i^{|\alpha|} \partial^{\alpha}\left(F_{\mathfrak{h}}{ }^{-1}(s) g\right) \\
& =\sum_{\alpha} \sum_{\mu} \sum_{\beta \leq \alpha} p_{\alpha} s_{\mu} i^{|\alpha|-|\mu|}\left(\begin{array}{c}
\alpha \\
\beta
\end{array}\right) \partial^{\beta}\left(x^{\mu}\right) \partial^{\alpha-\beta}(g) .
\end{aligned}
$$

But $\lim _{h \rightarrow 0} \partial^{\beta}\left(x^{\mu}\right)(h)=0$ unless $\beta=\mu$, hence

$$
\lim _{\substack{h \rightarrow 0 \\
h \in \Gamma}}\left[\partial(u) \cdot\left(F_{\mathfrak{h}}{ }^{-1}(s) g\right)\right](h)=\sum_{\alpha} \sum_{\mu \leq \alpha} p_{\alpha} s_{\mu} i^{|\alpha|-|\mu|}\left(\begin{array}{c}
\alpha \\
\mu
\end{array}\right) \mu ! \lim _{\substack{h \rightarrow 0 \\
h \in \Gamma}}\left[\partial^{\alpha-\mu}(g)\right](h) .
$$

On the other hand, we have

$$
\left\langle T_{\partial(s) \cdot p, \Gamma}, f\right\rangle=\lim _{\substack{h \rightarrow 0 \\ h \in \Gamma}}\left[\partial\left(F_{\mathfrak{h}}(\partial(s) \cdot p)\right) \cdot g\right](h) .
$$

Since $\partial(s) \cdot p=\sum_{\alpha} \sum_{\mu \leq \alpha} \frac{\alpha !}{(\alpha-\mu) !} s_{\mu} p_{\alpha} x^{\alpha-\mu}$, we obtain that

$$
\left\langle T_{\partial(s) \cdot p, \Gamma}, f\right\rangle=\sum_{\alpha} \sum_{\mu \leq \alpha} \frac{\alpha !}{(\alpha-\mu) !} s_{\mu} p_{\alpha} i^{|\alpha|-|\mu|} \lim _{\substack{h \rightarrow 0 \\ h \in \Gamma}}\left[\partial^{\alpha-\mu}(g)\right](h) .
$$

This proves the desired equality. 
Theorem 2.2. Let $p \in S\left(\mathfrak{h}^{*}\right)$ and $D \in \mathcal{D}(\mathfrak{g})^{G}$. Then, D.T $T_{p, \Gamma}=T_{\delta(D) . p, \Gamma}$.

Proof. Since $T_{p, \Gamma}$ is $G_{0}$-invariant, we have $\mathcal{I} . T_{p, \Gamma}=0$. Let $P \in \mathbb{C}\left\langle S(\mathfrak{g})^{G}, S\left(\mathfrak{g}^{*}\right)^{G}\right\rangle$; by Lemma 2.1 and an obvious induction, we obtain that $P . T_{p, \Gamma}=T_{\delta(P) . p, \Gamma}$. The theorem then follows from Lemma 1.1

Recall, see Remark 1.6 that $\widehat{\Theta}_{u, \Gamma} \in \mathrm{Db}\left(\mathfrak{g}_{0}\right)_{S_{+}}^{G_{0}}$ is determined by a locally integrable function on $\mathfrak{g}_{0}$. We still denote this function by $\widehat{\Theta}_{u, \Gamma}$.

Lemma 2.3. ([5 Lemme 6.1.2]) There exists $c_{\Gamma} \in \mathbb{C}^{*}$, such that

$$
a_{\Delta_{I}^{+}}(h)\left|\operatorname{det} \operatorname{ad}_{\mathfrak{g}_{0} / \mathfrak{h}_{0}}(h)\right|^{\frac{1}{2}} \widehat{\Theta}_{F_{\mathfrak{h}}(p), \Gamma}(h)=c_{\Gamma} p(h)
$$

for all $p \in S\left(\mathfrak{h}^{*}\right)^{\varepsilon_{I}}$ and $h \in \mathfrak{h}_{0}^{\text {reg }}$.

Remark. In the notation of the lemma, if $u=F_{\mathfrak{h}}(p)$, the function $\tilde{u}(i h)$ of [5] is replaced here by $p(h)$ since we are using $e^{-i \kappa(x, y)}$ in the definition of the Fourier transform.

Theorem 2.4. Let $p \in S\left(\mathfrak{h}^{*}\right)^{\varepsilon_{I}}$. There exists a bijective map

$$
\rho: \mathcal{D}(\mathfrak{g})^{G} \cdot T_{p, \Gamma} \longrightarrow \mathcal{D}(\mathfrak{h})^{W} \cdot p, \quad \rho\left(D \cdot T_{p, \Gamma}\right)=\delta(D) \cdot p
$$

which, through $\delta$, yields an isomorphism

$$
\rho: A(\mathfrak{g}) \cdot T_{p, \Gamma} \stackrel{\sim}{\sim} \mathcal{D}(\mathfrak{h})^{W} \cdot p .
$$

Proof. We first need to show that $\rho$ is well defined. Let $D \in \mathcal{D}(\mathfrak{g})^{G}$; by Theorem 2.2 we have

$$
D \cdot T_{p, \Gamma}=T_{\delta(D) \cdot p, \Gamma}=\widehat{\Theta}_{F_{\mathfrak{h}}(\delta(D) \cdot p), \Gamma} \cdot
$$

Suppose that $D \cdot T_{p, \Gamma}=0$. Then, the analytic function associated to $T_{\delta(D) . p, \Gamma} \in$ $\mathrm{Db}\left(\mathfrak{g}_{0}\right)_{S_{+}}^{G_{0}}$ vanishes on $\mathfrak{h}_{0}^{\text {reg }}$. Notice that, since $\delta(D)$ is $W$-invariant, $\delta(D) . p \in$ $S\left(\mathfrak{h}^{*}\right)^{\varepsilon_{I}}$. Therefore Lemma 2.3 gives $\delta(D) \cdot p=0$ on $\mathfrak{h}_{0}^{\text {reg }}$. Thus $\delta(D) \cdot p=0$ on $\mathfrak{h}$ and $\rho$ is well defined.

Now, it follows easily from $(\dagger)$ that $\rho$ is a linear bijection. Since $\mathcal{I} \cdot T_{p, \Gamma}=0$, the last assertion is clear.

Recall that we denote by $V_{\chi} \subset \mathcal{H}^{b(\chi)}\left(\mathfrak{h}^{*}\right)$ a simple $W$-module in the class of $\chi \in W^{\curlywedge}$.

Corollary 2.5. Let $p \in S\left(\mathfrak{h}^{*}\right)^{\varepsilon_{I}}$ such that $\mathbb{C W}$.p is simple. Then there exists $\chi \in$ $W^{\wedge}$ such that $V_{\chi}^{\varepsilon_{I}} \neq 0$. We have

1. $\mathcal{D}(\mathfrak{g}) \cdot T_{p, \Gamma} \stackrel{\sim}{\sim} \mathcal{N}_{\chi}$ and $\mathcal{D}(\mathfrak{g})^{G} \cdot T_{p, \Gamma} \stackrel{\sim}{\longrightarrow} V^{\chi}$;

2. $\mathcal{D}(\mathfrak{g}) \cdot \Theta_{F_{\mathfrak{h}}(p), \Gamma} \stackrel{\sim}{\longrightarrow} \mathcal{M}_{\chi}$ and $\mathcal{D}(\mathfrak{g})^{G} \cdot \Theta_{F_{\mathfrak{h}}(p), \Gamma} \stackrel{\sim}{\longrightarrow}\left(V^{\chi}\right)^{F_{\mathfrak{h}}}{ }^{-1}$.

Proof. The first assertion follows from $\mathcal{H}\left(\mathfrak{h}^{*}\right) \cong \mathbb{C} W$. Then, 1 and 2 are consequences of $V^{\chi} \cong \mathcal{D}(\mathfrak{h})^{W}$.p, Lemma 1.3 and Theorem 2.4.

Remark 2.6. Let $\chi \in W^{\wedge}$ be such that $V_{\chi}^{\varepsilon_{I}} \neq 0$. It follows obviously from the previous corollary that

$$
\mathcal{N}_{\chi} \cong \mathcal{D}(\mathfrak{g}) \cdot T_{p, \Gamma}, \quad \mathcal{M}_{\chi} \cong \mathcal{D}(\mathfrak{g}) \cdot \Theta_{u, \Gamma}
$$

where $0 \neq p \in V_{\chi}^{\varepsilon_{I}} \subset \mathcal{H}^{b(\chi)}\left(\mathfrak{h}^{*}\right)^{\varepsilon_{I}}$ and $u=F_{\mathfrak{h}}(p) \in \mathcal{H}^{b(\chi)}(\mathfrak{h})^{\varepsilon_{I}}$. 


\section{The DeComposition of $\operatorname{Db}\left(\mathfrak{g}_{0}\right)_{S_{+}}^{G_{0}}$ And $\operatorname{Db}\left(\mathfrak{g}_{0}\right)_{n i l}^{G_{0}}$}

Fix a real form $\mathfrak{g}_{0}$ of $\mathfrak{g}$ and let $\left[\mathfrak{h}_{1}\right], \ldots,\left[\mathfrak{h}_{r}\right]$ be the conjugacy classes of Cartan subalgebras in $\mathfrak{g}_{0}$. For each $j=1, \ldots, r$ we denote by

$\mathfrak{h}_{j, \mathbb{C}}=\mathfrak{h}_{j} \otimes_{\mathbb{R}} \mathbb{C}, \quad W_{j}=W\left(\mathfrak{g}, \mathfrak{h}_{j, \mathbb{C}}\right), \quad \Delta_{I, j}^{+}$a set of positive imaginary roots,

$\varepsilon_{I, j}: W\left(\mathfrak{h}_{j}\right)=W\left(G_{0}, \mathfrak{h}_{j}\right) \rightarrow\{ \pm 1\}$ the imaginary signature associated to $\mathfrak{h}_{j}$.

For each $j$ we fix a connected component $\Gamma_{j}$ of $\mathfrak{h}_{j}^{\text {reg }}$. The results of $\$ 2$ then apply to $\mathfrak{h}_{0}=\mathfrak{h}_{j}, \Gamma=\Gamma_{j}$ etc.

Remark 3.1. Recall that the $\mathfrak{h}_{j, \mathbb{C}}$ are $G$-conjugate. Therefore, if $1 \leqslant j, k \leqslant r$, the algebras $\mathcal{D}\left(\mathfrak{h}_{j, \mathbb{C}}\right)^{W_{j}}$ and $\mathcal{D}\left(\mathfrak{h}_{k, \mathbb{C}}\right)^{W_{k}}$ are naturally isomorphic. Denote this isomorphism by $\gamma_{j k}$ and let $\delta_{j}$ be the Harish-Chandra isomorphism from $A(\mathfrak{g})$ onto $\mathcal{D}\left(\mathfrak{h}_{j, \mathbb{C}}\right)^{W_{j}}$. One can check that $\delta_{k}=\gamma_{j k} \circ \delta_{j}$. Therefore, we can choose an "abstract" Cartan subalgebra $\mathfrak{h}$ and identify $\delta_{j}$ with the homomorphism $\delta: \mathcal{D}(\mathfrak{g})^{G} \rightarrow$ $\mathcal{D}(\mathfrak{h})^{W}$, where $W=W(G, \mathfrak{h})$. Then, if $\chi \in W^{\curlywedge}$, we have an irreducible $W$-module $V_{\chi} \subset \mathcal{H}^{b(\chi)}\left(\mathfrak{h}^{*}\right)$ and a simple $\mathcal{D}(\mathfrak{h})^{W}$-module $V^{\chi}$.

For each $\chi \in W^{\curlywedge}$, choose a simple $W$-module $V_{\chi, j} \subset \mathcal{H}^{b(\chi)}\left(\mathfrak{h}_{j, \mathbb{C}}^{*}\right), V_{\chi, j} \cong V_{\chi}$. Write $V_{\chi, j}=V_{\chi, j}^{\varepsilon_{I, j}} \oplus E_{\chi, j}$ with $E_{\chi, j}$ stable under $W\left(\mathfrak{h}_{j}\right)$. Let $\left\{v_{\chi, j}^{k}\right\}_{1 \leqslant k \leqslant d(\chi)}$ be a basis of $V_{\chi, j}$ such that

$$
V_{\chi, j}^{\varepsilon_{I, j}}=\bigoplus_{k=1}^{n_{j}(\chi)} \mathbb{C} v_{\chi, j}^{k}, \quad E_{\chi, j}=\bigoplus_{k=n_{j}(\chi)+1}^{d(\chi)} \mathbb{C} v_{\chi, j}^{k}
$$

(hence $\left.n_{j}(\chi)=\operatorname{dim} V_{\chi}^{\varepsilon_{I, j}}\right)$.

Lemma 3.2. The $\mathcal{D}\left(\mathfrak{h}_{j, \mathbb{C}}\right)^{W_{j}}$-module $S\left(\mathfrak{h}_{j, \mathbb{C}}^{*}\right)^{\varepsilon_{I, j}}$ decomposes as

$$
S\left(\mathfrak{h}_{j, \mathbb{C}}^{*}\right)^{\varepsilon_{I, j}}=\bigoplus_{\chi \in W^{\wedge}} \bigoplus_{k=1}^{n_{j}(\chi)} \mathcal{D}\left(\mathfrak{h}_{j, \mathbb{C}}\right)^{W_{j}} \cdot v_{\chi, j}^{k}
$$

with $\mathcal{D}\left(\mathfrak{h}_{j, \mathbb{C}}\right)^{W_{j}} \cdot v_{\chi, j}^{k} \cong V^{\chi}$.

Proof. Clearly, we can drop the index $j$ and write $\mathfrak{h}_{0}=\mathfrak{h}_{j}, \mathfrak{h}=\mathfrak{h}_{j, \mathbb{C}}, v_{\chi}^{k}=v_{\chi, j}^{k}$ etc. Since $\mathcal{D}(\mathfrak{h})^{W} . v_{\chi}^{k} \subset S\left(\mathfrak{h}^{*}\right)^{\varepsilon_{I}}$ for $1 \leqslant k \leqslant n(\chi)=\operatorname{dim} V_{\chi}^{\varepsilon_{I}}$, one has

$$
S\left(\mathfrak{h}^{*}\right)^{\varepsilon_{I}} \supset \bigoplus_{\chi \in W^{\wedge} k=1} \bigoplus_{k=1}^{n(\chi)} \mathcal{D}(\mathfrak{h})^{W} . v_{\chi}^{k} .
$$

Recall from \$1 that $S\left(\mathfrak{h}^{*}\right)=\bigoplus_{\chi} S\left(\mathfrak{h}^{*}\right)[\chi]$ with $S\left(\mathfrak{h}^{*}\right)[\chi]=\bigoplus_{k=1}^{d(\chi)} \mathcal{D}(\mathfrak{h})^{W} . v_{\chi}^{k}$. Write $S\left(\mathfrak{h}^{*}\right)[\chi]=E_{1} \oplus E_{2}$, where $E_{1}=\bigoplus_{k=1}^{n(\chi)} \mathcal{D}(\mathfrak{h})^{W} . v_{\chi}^{k}$ and $E_{2}=\bigoplus_{k=n(\chi)+1}^{d(\chi)} \mathcal{D}(\mathfrak{h})^{W} \cdot v_{\chi}^{k}$. Notice that $E_{1}, E_{2}$ are stable under $W\left(\mathfrak{h}_{0}\right)$ and that we have $S\left(\mathfrak{h}^{*}\right)[\chi]^{\varepsilon_{I}}=E_{1} \oplus E_{2}^{\varepsilon_{I}}$.

We now show that $E_{2}^{\varepsilon_{I}}=0$. This will prove that

$$
S\left(\mathfrak{h}^{*}\right)^{\varepsilon_{I}}=\bigoplus_{\chi \in W^{\wedge}} \bigoplus_{k=1}^{n(\chi)} \mathcal{D}(\mathfrak{h})^{W} \cdot v_{\chi}^{k} .
$$

Let $D \in \mathcal{D}(\mathfrak{h})^{W}$ and $v \in V_{\chi}$. Notice first that if $D . v \neq 0$, the operator $D$ yields an isomorphism of $W$-modules $V_{\chi} \simeq D$. $V_{\chi}$. Therefore, if $V_{\chi}=\bigoplus_{k} S_{k}$ with an 
$S_{k}$ irreducible $W\left(\mathfrak{h}_{0}\right)$-module, we get that $D . V_{\chi}=\bigoplus_{k} D . S_{k}, D . S_{k} \cong S_{k}$. It follows that if $v \in E_{\chi}$ (the $W\left(\mathfrak{h}_{0}\right)$-stable complement of $\left.V_{\chi}^{\varepsilon_{I}}\right)$, then D.v $\in$ D.E $E_{\chi}$ with $D . E_{\chi} \cap S\left(\mathfrak{h}^{*}\right)^{\varepsilon_{I}}=0$. Let $p=\sum_{k=n(\chi)+1}^{d(\chi)} D_{k} \cdot v_{\chi}^{k} \in E_{2}$. Then, $\mathbb{C} W\left(\mathfrak{h}_{0}\right) \cdot p \subset$ $\sum_{k>n(\chi)} \mathbb{C} W\left(\mathfrak{h}_{0}\right) \cdot\left(D_{k} \cdot v_{\chi}^{k}\right)$ and, by the previous remarks, $\left(\mathbb{C} W\left(\mathfrak{h}_{0}\right) \cdot\left(D_{k} \cdot v_{\chi}^{k}\right)\right)^{\varepsilon_{I}}=0$. Thus $\left(\mathbb{C} W\left(\mathfrak{h}_{0}\right) \cdot p\right)^{\varepsilon_{I}}=0$, which shows that $E_{2}^{\varepsilon_{I}}=0$.

Recall the following result:

Proposition 3.3 ([5 Proposition 6.1.1]). (1) The linear map

$$
\mathbf{T}: \bigoplus_{j=1}^{r} S\left(\mathfrak{h}_{j, \mathbb{C}}^{*}\right)^{\varepsilon_{I, j}} \longrightarrow \mathrm{Db}\left(\mathfrak{g}_{0}\right)_{S_{+}}^{G_{0}}, \quad \mathbf{T}\left(p_{1}, \ldots, p_{r}\right)=\sum_{j=1}^{r} T_{p_{j}, \Gamma_{j}}
$$

is an isomorphism of vector spaces.

(2) The map $\mathbf{T}$ induces an isomorphism:

$$
\bigoplus_{j=1}^{r} \mathcal{H}\left(\mathfrak{h}_{j, \mathbb{C}}^{*}\right)^{\varepsilon_{I, j}} \stackrel{\sim}{\longrightarrow}\left\{T \in \mathrm{Db}\left(\mathfrak{g}_{0}\right)_{S_{+}}^{G_{0}}: S_{+}(\mathfrak{g})^{G} \cdot T=0\right\} .
$$

Proof. (2) follows from the proof of [5, Proposition 6.1.1].

Theorem 3.4. Set $\mathbf{T}\left(\mathfrak{h}_{j}\right)=\sum_{p \in S\left(\mathfrak{h}_{j, \mathbb{C}}^{*}\right)^{\varepsilon}, j} \mathbb{C} T_{p, \Gamma_{j}}$. Then we have the following decomposition of $\mathcal{D}(\mathfrak{g})^{G}$-modules:

$$
\mathrm{Db}\left(\mathfrak{g}_{0}\right)_{S_{+}}^{G_{0}}=\bigoplus_{j=1}^{r} \mathbf{T}\left(\mathfrak{h}_{j}\right)
$$

with

$$
\mathbf{T}\left(\mathfrak{h}_{j}\right)=\bigoplus_{\chi \in W^{\wedge}} \bigoplus_{k=1}^{n_{j}(\chi)} \mathcal{D}(\mathfrak{g})^{G} \cdot T_{v_{\chi, j}^{k}, \Gamma_{j}}
$$

and $\mathcal{D}(\mathfrak{g})^{G} \cdot T_{v_{\chi, j}^{k}, \Gamma_{j}} \cong \mathcal{N}_{\chi}^{G}$.

Proof. The decomposition of $\mathbf{T}\left(\mathfrak{h}_{j}\right)$, as a $\mathcal{D}(\mathfrak{g})^{G}$-module, is consequence of Theorem [2.4 Lemma 3.2 (using the isomorphism $\delta_{j}: A(\mathfrak{g}) \stackrel{\sim}{\longrightarrow}\left(\mathfrak{h}_{j, \mathbb{C}}\right)^{W_{j}}$ ) and Proposition 3.3 The decomposition of $\operatorname{Db}\left(\mathfrak{g}_{0}\right)_{S_{+}}^{G_{0}}$ follows from Proposition 3.3 ,

Using the Fourier transform, we obtain the following:

Corollary 3.5. The $\mathcal{D}(\mathfrak{g})^{G}$-module $\mathrm{Db}\left(\mathfrak{g}_{0}\right)_{\text {nil }}^{G_{0}}$ decomposes as

$$
\mathrm{Db}\left(\mathfrak{g}_{0}\right)_{n i l}^{G_{0}}=\bigoplus_{j=1}^{r} \bigoplus_{\chi \in W^{\wedge}} \bigoplus_{k=1}^{n_{j}(\chi)} \mathcal{D}(\mathfrak{g})^{G} \cdot \Theta_{F_{\mathfrak{h}}-1}\left(v_{\chi, j}^{k}\right), \Gamma_{j}
$$

with $\mathcal{D}(\mathfrak{g})^{G} \cdot \Theta_{F_{\mathfrak{h}}{ }^{-1}\left(v_{\chi, j}^{k}\right), \Gamma_{j}} \cong \mathcal{M}_{\chi}^{G}$.

The next corollary follows from Theorem 3.4 and Corollary 3.5 .

Corollary 3.6. We have

$$
\mathrm{Db}\left(\mathfrak{g}_{0}\right)_{S_{+}}^{G_{0}} \cong \bigoplus_{\chi \in W^{\wedge}} m_{\chi} \mathcal{N}_{\chi}^{G}, \quad \operatorname{Db}\left(\mathfrak{g}_{0}\right)_{n i l}^{G_{0}} \cong \bigoplus_{\chi \in W^{\wedge}} m_{\chi} \mathcal{M}_{\chi}^{G}
$$

where $m_{\chi}=\sum_{j=1}^{r} \operatorname{dim} V_{\chi}^{\varepsilon_{I, j}}$. 
Remark 3.7. Let $\chi \in W^{\curlywedge}$. It is not always possible to "realize" the modules $\mathcal{N}_{\chi}$ and $\mathcal{M}_{\chi}$ as $\mathcal{D}(\mathfrak{g}) . T$ for some $T \in \operatorname{Db}\left(\mathfrak{g}_{0}\right)$, where $\mathfrak{g}_{0}$ is a real form of $\mathfrak{g}$. By the previous results, this statement is equivalent to the existence of a Cartan subalgebra $\mathfrak{h}_{j} \subset \mathfrak{g}_{0}$ such that $V_{\chi}^{\varepsilon_{I, j}} \neq 0$. D. Renard has observed that, using the results of W. Rossmann [15], this can be translated to a question about centralizers of nilpotent elements. Fix a real form $\mathfrak{g}_{\mathbb{R}}$ of $\mathfrak{g}$ with adjoint group $G_{\mathbb{R}}$. If $x \in \mathfrak{g}_{\mathbb{R}}$ is nilpotent one defines a subgroup of the component group $A(G . x)$ (see $\$$ for notation) by

$$
A\left(G_{\mathbb{R}} \cdot x\right)=G_{\mathbb{R}}^{x} / G_{\mathbb{R}}^{x} \cap\left(G^{x}\right)^{0} .
$$

Recall that $\chi \in W^{\wedge}$ can be written $\sigma(\mathbf{O}, \psi)$ via the Springer correspondence, where $\mathbf{O} \subset \mathfrak{g}$ is a nilpotent orbit and $\psi: A(\mathbf{O}) \rightarrow \mathrm{GL}(E)$ is an irreducible representation. Then, by [15. Corollary $3.2 \&$ Theorem 3.3], there exists a Cartan subalgebra $\mathfrak{h}_{0} \subset \mathfrak{g}_{\mathbb{R}}$ such that $V_{\chi}^{\varepsilon_{I}} \neq 0$ if, and only if, there exists a nilpotent element $x \in \mathfrak{g}_{\mathbb{R}}$ such that $\mathbf{O}=G \cdot x$ and $E^{A\left(G_{\mathbb{R}} \cdot x\right)} \neq 0$.

Let $\mathfrak{g}=\mathfrak{s p}(\ell, \mathbb{C})$ and let $\phi \in W^{\wedge}$ be the long sign character, i.e. $V_{\phi}=\mathbb{C} \pi_{l}$ where $\pi_{l}$ is the product of the long roots. Then, see [6, §13.3], $\phi=\sigma(\mathbf{O}, \psi)$ where $\mathbf{O}=G . x$ is the subregular nilpotent orbit with partition $[2 \ell-2,2]$ and $\psi$ is the nontrivial character of $A(\mathbf{O}) \cong\{ \pm 1\}$. The real forms of $\mathfrak{g}$ are $\mathfrak{s p}(\ell, \mathbb{R})$ and the $\mathfrak{s} p(p, q)$, $p+q=\ell$. Assume now that $\ell \geqslant 3$. By the classification of nilpotent orbits in $\mathfrak{s p}(p, q)$, see [7. Theorem 9.2.5], we know that $\mathbf{O} \cap \mathfrak{s p}(p, q)=\emptyset$. Hence, by Rossmann's results, $V_{\phi}^{\varepsilon_{I, j}}=0$ for each Cartan subalgebra $\mathfrak{h}_{j} \subset \mathfrak{s p}(p, q)$. On the other hand, if $G_{\mathbb{R}}$ is the adjoint group of $\mathfrak{s p}(\ell, \mathbb{R})$, one can show that $A\left(G_{\mathbb{R}} \cdot x\right)=A(G \cdot x)$. Thus, with the above notation, $E^{A\left(G_{\mathbb{R}} \cdot x\right)}=0$ and it follows that $V_{\phi}^{\varepsilon_{I, j}}=0$ for each Cartan subalgebra $\mathfrak{h}_{j} \subset \mathfrak{s p}(\ell, \mathbb{R})$. For instance, when $\mathfrak{g}=\mathfrak{s p}(3, \mathbb{R})$ there are six conjugacy classes of Cartan subalgebras and one can directly verify (without using [15]) that $V_{\phi}^{\varepsilon_{I, j}}=0$ for $j=1, \ldots, 6$. We thank D. Renard for showing this computation to us.

Let $x \in \mathbf{N}\left(\mathfrak{g}_{0}\right)$ and denote by $\beta_{x}$ the Liouville (Kostant-Kirillov) measure on $G_{0} . x$. By [14] one can define $\Theta_{x} \in \mathrm{Db}\left(\mathfrak{g}_{0}\right)_{n i l}^{G_{0}}$ by $\left\langle\Theta_{x}, f\right\rangle=\int_{G_{0} . x} f d \beta_{x}$ for all $f \in \mathcal{C}_{c}^{\infty}\left(\mathfrak{g}_{0}\right)$. Set $\mathbf{O}=$ G.x. Then, see [9], 10] or [18; $\Theta_{x}$ is homogeneous of degree $\lambda_{\mathbf{O}}=\frac{1}{2} \operatorname{dim} \mathbf{O}-\operatorname{dim} \mathfrak{g}$ and satisfies

$$
\mathcal{D}(\mathfrak{g}) \cdot \Theta_{x} \cong \mathcal{M}_{\chi \mathrm{o}}
$$

for some $\chi_{\mathbf{O}} \in W^{\wedge}$ such that $\lambda_{\mathbf{O}}=\nu-n-b\left(\chi_{\mathbf{O}}\right)$.

Corollary 3.8. There exists $j \in\{1, \ldots, r\}$ and $u \in{F_{\mathfrak{h}}}^{-1}\left(V_{\chi_{\mathbf{o}}, j}\right)^{\varepsilon_{I, j}}$ such that

$$
\mathcal{D}(\mathfrak{g})^{G} \cdot \Theta_{x} \cong \mathcal{D}(\mathfrak{g})^{G} \cdot \Theta_{u, \Gamma_{j}} .
$$

Proof. Since $\mathcal{D}(\mathfrak{g})^{G} \cdot \Theta_{x} \cong \mathcal{M}_{\chi_{\mathrm{O}}}^{G}$ is a simple submodule of $\mathrm{Db}\left(\mathfrak{g}_{0}\right)_{n i l}^{G_{0}}$, the claim follows from Corollary 3.5 .

Remark 3.9. It is proved in [1], see also [5], that $\Theta_{x}$ can be written as $\sum_{j=1}^{r} \Theta_{a_{j}, \Gamma_{j}}$ with $a_{j} \in \mathcal{H}^{b\left(\chi_{0}\right)}\left(\mathfrak{h}_{j, \mathbb{C}}\right)^{\varepsilon_{I, j}}$. It is easily seen that we may assume $\mathbb{C} W . a_{j} \cong V_{\chi_{\mathrm{o}}}$ for all $j$ such that $a_{j} \neq 0$. W. Rossmann [15] has given conditions to ensure that $\Theta_{x}=\Theta_{a_{j}, \Gamma_{j}}$ for some $j$. 


\section{EXAMPLE: THE COMPLEX CASE}

We assume in this section that $\mathfrak{g}_{0}=\mathfrak{g}_{1}^{\mathbb{R}}$ is a complex semisimple Lie algebra, $\mathfrak{g}_{1}$, viewed as a real Lie algebra. Then, $\mathfrak{g}$ can be identified with $\mathfrak{g}_{1} \times \mathfrak{g}_{1}$ and $\mathfrak{g}_{0}$ with the diagonal $\left\{(a, a) \in \mathfrak{g}_{1} \times \mathfrak{g}_{1}\right\}$. Let $\mathfrak{h}_{1}$ be a Cartan subalgebra of $\mathfrak{g}_{1}$. Recall the following well-known facts, see [17] or [18:

. $\mathfrak{h}_{0}=\left\{(a, a): a \in \mathfrak{h}_{1}\right\}$ is a Cartan subalgebra of $\mathfrak{h}_{0}$ and $\mathfrak{h}=\mathfrak{h}_{0} \otimes_{\mathbb{R}} \mathbb{C}=\mathfrak{h}_{1} \times \mathfrak{h}_{1}$;

. $W(\mathfrak{g}, \mathfrak{h})=W_{1} \times W_{1}$, where $W_{1}=W\left(\mathfrak{g}_{1}, \mathfrak{h}_{1}\right)$, and $W\left(\mathfrak{h}_{0}\right)=\{(w, w) \in W\}$ is isomorphic to $W_{1}$;

- there is a unique conjugacy class $\left[\mathfrak{h}_{0}\right]$ of Cartan subalgebras and $\mathfrak{h}_{0}^{\prime}$ is connected;

. the roots in $\Delta(\mathfrak{g}, \mathfrak{h})$ are complex and, therefore, $\varepsilon_{I}=1$;

- the irreducible representations of $W$ are of the form $\chi=\phi \otimes \mu, \phi, \mu \in W_{1}$;

- one has $\phi=\phi^{*}$ for all $\phi \in W_{1}^{\hat{\text { }}}$, where $\phi^{*}$ is the dual representation.

Observe that $\mathcal{D}(\mathfrak{g})=\mathcal{D}\left(\mathfrak{g}_{1}\right) \otimes \mathcal{D}\left(\mathfrak{g}_{1}\right)$ and $\mathcal{D}(\mathfrak{g})^{G}=\mathcal{D}\left(\mathfrak{g}_{1}\right)^{G_{1}} \otimes \mathcal{D}\left(\mathfrak{g}_{1}\right)^{G_{1}}$.

Lemma 4.1. Let $\chi \in W^{\curlywedge}$. Then, the simple $\mathcal{D}(\mathfrak{g})$-module $\mathcal{M}_{\chi}$ is of the form $\mathcal{M}_{\phi} \otimes \mathcal{M}_{\mu}$ for some $\phi, \mu \in W_{1}^{\wedge}$.

Proof. The claim follows easily from the definition of the category $\mathrm{C}(\mathcal{M})$ and the decomposition of the $W$-module $S\left(\mathfrak{h}^{*}\right)=S\left(\mathfrak{h}_{1}^{*}\right) \otimes S\left(\mathfrak{h}_{1}^{*}\right)$.

Corollary 4.2. ([18, Theorem 6.11]) We have

$$
\mathrm{Db}\left(\mathfrak{g}_{0}\right)_{n i l}^{G_{0}} \cong \bigoplus_{\phi \in W_{1}^{\wedge}} \mathcal{M}_{\phi}^{G_{1}} \otimes \mathcal{M}_{\phi}^{G_{1}}
$$

as a $\mathcal{D}(\mathfrak{g})^{G}$-module.

Proof. Let $\chi=\phi \otimes \mu \in W^{\curlywedge}$. Then, $V_{\chi}^{\varepsilon_{I}}=\left(V_{\phi} \otimes V_{\mu}\right)^{W_{1}} \neq 0$ if, and only if, $\phi=\mu$ and therefore $n(\chi)=1$. The assertion now follows from Corollary 3.5.

Recall the following general results from [13. Since the module $\mathcal{M}_{\chi}$ is irreducible and $G$-equivariant, its support is the closure of a nilpotent orbit $\mathbf{O}=G . x$. Furthermore, if $\imath: \mathbf{O} \hookrightarrow \mathfrak{g}$ is the inclusion, $\mathcal{M}_{\chi}$ is uniquely determined by its $(D-$ module) inverse image $\mathcal{L}_{\chi}:=\imath^{!} \mathcal{M}_{\chi}$. The $\mathcal{D}_{\mathbf{O}}$-module $\mathcal{L}_{\chi}$ is an irreducible integrable connection associated to an irreducible representation $\psi$ of the component group $A(\mathbf{O}):=G^{x} /\left(G^{x}\right)^{0}$ (where $\left(G^{x}\right)^{0}$ is the connected component of the centralizer $\left.G^{x}\right)$. Therefore, since $\chi$ is uniquely determined by $\mathbf{O}$ and $\psi$, we set $\chi=\sigma(\mathbf{O}, \psi)$.

In our situation, i.e. in the complex case, we have $\mathbf{O}=\mathbf{O}_{1}^{1} \times \mathbf{O}_{1}^{2}$ with $\mathbf{O}_{1}^{j}$ nilpotent orbits in $\mathfrak{g}_{1}$ for $j=1,2$. Then, $\chi=\sigma(\mathbf{O}, \psi)=\phi_{1} \otimes \phi_{2}, \mathcal{L}_{\chi}=\mathcal{L}_{\phi_{1}} \otimes \mathcal{L}_{\phi_{2}}$, $\phi_{j}=\sigma\left(\mathbf{O}_{1}^{j}, \psi_{j}\right), \psi=\psi_{1} \otimes \psi_{2}$. Note that $b(\chi)=b\left(\phi_{1}\right)+b\left(\phi_{2}\right)$ and $\lambda_{\mathbf{O}}=\lambda_{\mathbf{O}_{1}^{1}}+\lambda_{\mathbf{O}_{1}^{2}}$.

Let $x \in \mathbf{N}\left(\mathfrak{g}_{0}\right)$; set $x=\left(x_{1}, x_{1}\right), x_{1} \in \mathbf{N}\left(\mathfrak{g}_{1}\right), \mathbf{O}_{1}=G_{1} \cdot x_{1}, \mathbf{O}=G . x=\mathbf{O}_{1} \times \mathbf{O}_{1}$. The inclusion $\imath: \mathbf{O} \hookrightarrow \mathfrak{g}$ is equal to $\imath_{1} \times \imath_{1}$, where $\imath_{1}: \mathbf{O}_{1} \hookrightarrow \mathfrak{g}_{1}$. By (3.1) and Corollary 4.2 there exist $\chi \in W^{\curlywedge}, \chi_{1} \in W_{1}^{\widehat{s}}$ such that $\chi=\chi_{1} \otimes \chi_{1}$ and $\mathcal{D}(\mathfrak{g}) \cdot \Theta_{x} \cong \mathcal{M}_{\chi_{1}} \otimes \mathcal{M}_{\chi_{1}}$.

It is known (Harish-Chandra) that $\Theta_{x}=\Theta_{u, \mathfrak{h}_{0}^{\prime}}$ for some $u \in S\left(\mathfrak{h}_{1}\right) \otimes S\left(\mathfrak{h}_{1}\right)$. The following result has been proved by various authors; see [2, 3] (when $\mathbf{O}_{1}$ is "special"), 8], 9], [16.

Theorem 4.3. One has $\chi_{1}=\sigma\left(\mathbf{O}_{1}\right.$, triv), and there exists $p \in\left(V_{\chi_{1}} \otimes V_{\chi_{1}}\right)^{W_{1}}$ such that $\Theta_{x}=\Theta_{F_{\mathfrak{h}}(p), \mathfrak{h}_{0}^{\prime}}$. 
Proof. Recall from [9] or [10] that $\chi=\chi_{1} \otimes \chi_{1}=\sigma(\mathbf{O}$, triv). This means that

$$
\mathcal{L}_{\chi}=\mathcal{L}_{\chi_{1}} \otimes \mathcal{L}_{\chi_{1}}=\mathcal{O}_{\mathbf{O}}=\mathcal{O}_{\mathbf{O}_{1}} \otimes \mathcal{O}_{\mathbf{O}_{1}}
$$

(where we denote by $\mathcal{O}_{X}$ the structural sheaf of an algebraic variety $X$ ). This yields $\mathcal{L}_{\chi_{1}}=\mathcal{O}_{\mathbf{O}_{1}}$ and $\chi_{1}=\sigma\left(\mathbf{O}_{1}\right.$, triv $)$.

Set $T_{x}=\widehat{\Theta}_{x} ;$ then $\mathcal{D}(\mathfrak{g}) \cdot T_{x}=\mathcal{N}_{\chi_{1}} \otimes \mathcal{N}_{\chi_{1}}$ (see Lemma 1.4). Since $S_{+}\left(\mathfrak{g}^{*}\right)^{G} \cdot \Theta_{x}=0$ we have $S_{+}(\mathfrak{g})^{G} \cdot T_{x}=0$. It follows from Proposition 3.3(2) that we can write $T_{x}=T_{p, \mathfrak{h}_{0}^{\prime}}$ for some $p \in\left(\mathcal{H}\left(\mathfrak{h}_{1}^{*}\right) \otimes \mathcal{H}\left(\mathfrak{h}_{1}^{*}\right)\right)^{W_{1}}$ or, equivalently, $\Theta_{x}=\Theta_{F_{\mathfrak{h}}(p), \mathfrak{h}_{0}^{\prime}}$. Now, by Theorem 2.4 $\mathcal{D}(\mathfrak{h})^{W} . p=V^{\chi_{1}} \otimes V^{\chi_{1}}$ and therefore $\mathbb{C} W \cdot p \cong V_{\chi_{1}} \otimes V_{\chi_{1}}$. Moreover, $T_{x}=T_{p, \mathfrak{h}_{0}^{\prime}}$ is homogeneous of degree $b\left(\chi_{\mathbf{O}}\right)-2 \nu=2 b\left(\chi_{1}\right)-2 \nu=\operatorname{deg} p-2 \nu$. Thus $\operatorname{deg} p=2 b\left(\chi_{1}\right)$ and, by definition of $V_{\chi_{1}}, p \in\left(V_{\chi_{1}} \otimes V_{\chi_{1}}\right)^{W_{1}}$.

\section{ACKNOWLEDGMENT}

This work was partially completed while the author was visiting the Weizmann Institute of Science. He thanks this institution, and A. Joseph, for their hospitality.

\section{REFERENCES}

1. J. Arthur, The $L^{2}$-Lefschetz numbers of Hecke operators, Invent. Math., 97 (1989), 257-290. MR 91i:22024

2. D. Barbasch and D. Vogan, The local structure of characters, J. Funct. Anal., 37 (1980), 27-55. MR 82e:22024

3. (1982), 153-199. MR 83m:22026

4. (1983), 350-382. MR 84h:22038

5. A. Bouaziz, Intégrales orbitales sur les algèbres de Lie réductives, Invent. Math., 115 (1994), 163-207. MR 95a:22017

6. R. W. Carter, Finite Groups of Lie Type, Conjugacy Classes and Complex Characters, John Wiley \& Sons, Chichester, 1985. MR 94k:20020

7. D. H. Collingwood and W. M. McGovern, Nilpotent Orbits in Semisimple Lie Algebras, Van Nostrand, New York, 1993. MR 94j:17001

8. V. Ginsburg, Intégrales sur les orbites nilpotentes et représentations des groupes de Weyl, C. R. Acad. Sci. Paris, 296 (1983), 249-252. MR 85b:22019

9. R. Hotta and M. Kashiwara, The invariant holonomic system on a semisimple Lie algebra, Invent. Math., 75 (1984), 327-358. MR 87i:22041

10. T. Levasseur, Equivariant D-modules attached to nilpotent orbits in a semisimple Lie algebra, to appear in Transformation Groups, (1998).

11. T. Levasseur and J. T. Stafford, Invariant differential operators and an homomorphism of Harish-Chandra, J. Amer. Math. Soc., 8 (1995), 365-372. MR 2001b:16025

12. The kernel of an homomorphism of Harish-Chandra, Ann. Sci. Éc. Normale Sup., 29 (1996), 385-397. MR 97b:22019

13. L Semi-simplicity of invariant holonomic systems on a reductive Lie algebra, Amer. J. Math., 119 (1997), 1095-1117. MR 99g:17020

14. R. Rao, Orbital integrals on reductive groups, Ann. Math., 96 (1972), 505-510.

15. W. Rossmann, Nilpotent Orbital Integrals in a Real Semisimple Lie Algebra and Representations of Weyl Groups, in Actes du colloque en l'honneur de Jacques Dixmier, Progress in Math. 92, Birkhäuser, Boston, 1990, 333-397. MR 92c:22022

16. Invariant Eigendistributions on a Semisimple Lie Algebra and Homology Classes on the Conormal Variety. II. Representations of Weyl Groups, J. Funct. Anal., 96 (1991), 155-193. MR 92g:22034 
17. V. S. Varadarajan, Harmonic Analysis on Real Reductive Groups, Part I, Lecture Notes in Mathematics 576, Springer-Verlag, Berlin/New York, 1977. MR 57:12789

18. N. Wallach, Invariant differential operators on a reductive Lie algebra and Weyl group representations, J. Amer. Math. Soc., 6 (1993), 779-816. MR 94a:17014

Département de Mathématiques, Université de Brest, 29285 Brest, France

E-mail address: Thierry.Levasseur@univ-brest.fr

URL: http://maths2.univ-brest.fr/ levasseu 